\title{
Three-Dimensional Super-Resolution Imaging of Thick Biological Samples
}

\author{
A. Vaziri, J. Tang, H. Shroff and C. Shank \\ Janelia Farm Research Campus, Howard Hughes Medical Institute, Ashburn, VA 20147
}

Recent advances in optical microscopy have created the capability of creating images in biological samples beyond the diffraction limit at nanometer resolution [1-9]. A general feature of most of the techniques based on photoactivated localization microscopy (PALM) [1,9] or stochastic optical reconstruction microscopy (STORM) [3] has been the use of thin biological samples and a sample geometry using total internal reflection that limits the imaging depth to a fraction of an optical wavelength. However, in order to study whole cells or organelles which are typically up to $\sim 15 \mu \mathrm{m}$ deep into the cell, the extension of these methods to a 3D super-resolution technique is required.

Initial studies, based on defocusing [10], astigmatism [5] and other [11], have extended superresolution imaging to $3 \mathrm{D}$, however only up to a few hundreds on nanometer in depth in biological samples. Thus, super-resolution imaging would benefit greatly by adopting a widefield technique and combining it with optical sectioning for enhanced signal to noise ratio at depth.

PALM is a recently developed super-resolution technique based on photoswitchable fluorescent molecules. In PALM numerous sparse subsets of photoactivatable fluorescent proteins are activated, localized and then bleached. The aggregated position information from all subsets is then assembled into a super-resolution image.

Temporal focusing $[12,13]$ is a technique which provides optical sectioning comparable to a confocal microscope in a widefield geometry. This is experimentally achieved by an appropriate dispersion compensation scheme which selectively compensates the dispersion of a broadened ultra short pulse only in a single imaging plane. Compared with an epi-fluorescence technique, this minimum width results in a depth of field that is orders of magnitude smaller

In this work we have used temporal focusing to selectively activate a thin layer of photoactivatable fluorescent proteins at different layers several microns deep in a cellular sample. This thin layer is then excited and imaged using the PALM technique with a demonstrated resolution of better than $50 \mathrm{~nm}$. A succession of thin layer images was then combined to produce a volume image several microns in depth.

We have generated super-resolution images over an axial range of $\sim 10 \mu \mathrm{m}$ in both mitochondriallylabeled fixed cells, and in the membranes of living S2 Drosophila cells. Fig.1 shows a multilayer super-resolution image of mitochondrial network over $5 \mu \mathrm{m}$ using a color coding to represent depth information

Given the fact that temporal focusing makes optical sectioning over a range of many tens of microns possible, this technique can be expected to become a method of choice when single-molecule 
resolution in larger volumes is required. Areas which could benefit from temporal focusing-PALM include developmental biology on molecular level or in neuroscience studies using drosophila where questions of neural circuitry could be addressed on the level of whole brains.

\section{References}

[1] Betzig, E., et al., Science, 2006. 313(5793): p. 1642-1645.

[2] Wang, Y.-1., et al., J. Cell Biol., 2006. 174(4): p. 481-484.

[3] Rust, M.J., M. Bates, and X. Zhuang, Nat. Methods, 2006. 3(10): p. 793-796.

[4] Gustafsson, M.G.L., et al., Biophysical Journal, 2008. 94(12): p. 4957-4970.

[5] Huang, B.W., W. and M.Z. Q.Bates, X. W., Science, 2008. 319(5864): p. 810-813.

[6] Huisken, J., et al., Science, 2004. 305(5686): p. 1007-9.

[7]Willig, K.I., et al., Nature, 2006. 440(7086): p. 935-939.

[8] Donnert, G., et al., PNAS, 2006. 103: p. 11440-11445.

[9] Hess, S.T., T.P.K. Girirajan, and M.D. Mason, Biophys. J., 2006. 91(11): p. 4258-4272.

[10] Juette, M.F., et al., Nature Methods, 2008. 5(6): p. 527-529.

[11] Schmidt, R., et al., Nature Methods, 2008. 5(6): p. 539-544.

[12] Oron, D., E. Tal, and Y. Silberberg, Optics Express, 2005. 13(5): p. 1468-1476.

[13] Zhu, G.H., et al., Optics Express, 2005. 13(6): p. 2153-2159.
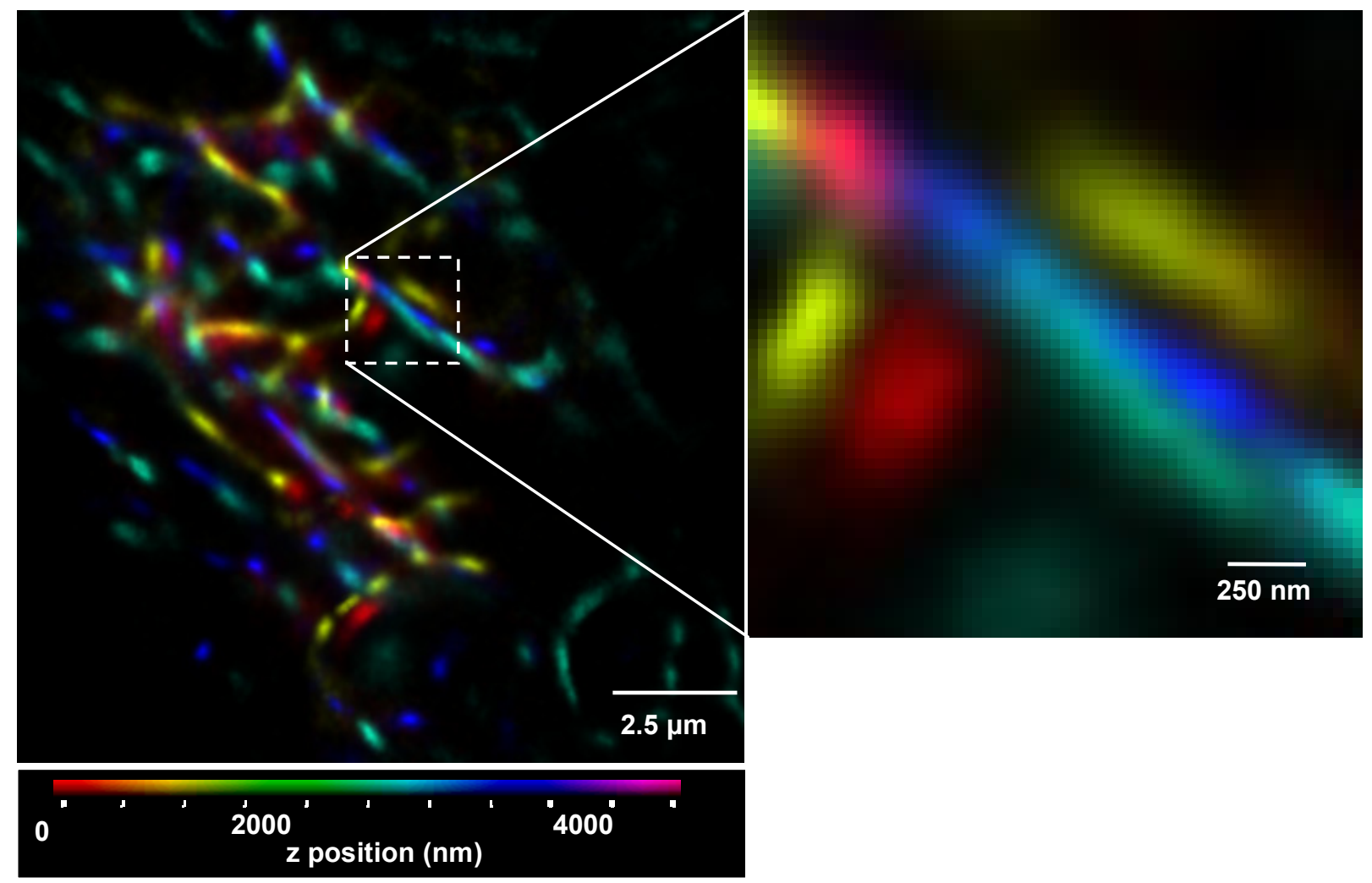

FIG. 1. Multilayer super-resolution imaging of mitochondrial network over $5 \mu \mathrm{m}$ in depth 